\title{
Balance de Energía y Exergía de un Horno de Cuba Vertical para la Producción de Cal
}

\section{Energy Balance and Exergy of a Vertical Cell Furnace for Lime Production}

DOI: https://doi.org/10.17981/ijmsor.04.01.08

Research Article - Reception Date: jun 15, 2019-Acceptance Date: Sep 25, 2019

\author{
Alexis Sagastume Gutiérrez ${ }^{1}$ and Juan B. Cogollos Martínez ${ }^{2}$ \\ Universidad de la Costa. Barranquilla (Colombia) ${ }^{1}$ \\ Universidad de Cienfuegos. Cienfuegos (Cuba) ${ }^{2}$ \\ asagastu1@cuc.edu.co
}

To reference this paper:

Sagastume Gutiérrez and J. Cogollos Martínez "Balance de energía y exergía de un horno de cuba vertical para la producción de cal”, IJMSOR, vol. 4, no. 1, 2019. https://doi. org/10.17981/ijmsor.04.01.08

Resumen-- El trabajo trata las metodologías y los indicadores para la evaluación de los impactos de uso del agua dulce, los métodos existentes analizan fundamentalmente la cantidad de agua usada con relación a los impactos ocasionados. Teniendo en cuenta que hay una necesidad reconocida para considerar los impactos, específicamente sobre bases del ciclo de vida, la problemática es que se consideran insuficientes los datos o no son confiables en las bases de datos del ciclo de vida del uso del agua, además no coincide el método de valoración del impacto del ciclo de vida para lo relacionado con los impactos estimados en el uso del agua dulce, se resaltan estas dificultades y se analizan las ventajas, limitaciones, diferencias en los resultados entre diversos métodos y se observa la necesidad de una metodología perfeccionada para la evaluación de los impactos del uso del agua sobre bases del ciclo de vida. La producción de cal viva es un alto consumidor de energía que además se caracteriza por elevadas emisiones de CO2. Este trabajo se propone desarrollar el balance energético y exergético del proceso de calcinación de calizas con el objetivo de identificar los factores más influyentes en el consumo de combustible. Los resultados muestran que la destrucción de exergía durante debido a la combustión del combustible y a la transferencia de calor y momento del proceso son los procesos más irreversibles. Los resultados además muestran que la energía y la exergía contenida en los gases de escape representan la principal pérdida del proceso. La combinación de estos factores representa más del $50 \%$ de la energía suministrada al proceso.

Palabras clave-- Energía; exergía; eficiencia

\begin{abstract}
This paper aims with methodologies and indicators for evaluating the impacts of freshwater use, the existing methods fundamentally analyze the amount of water used in relation to the impacts caused. Taking into account that there is a recognized need to consider the impacts, specifically on life cycle bases, the problem is that the data is considered insufficient or unreliable in the life cycle databases of water use, in addition not The method for evaluating the impact of the life cycle coincides with regard to the estimated impacts on the use of fresh water. These difficulties are highlighted and the advantages, limitations, differences in the results between different methods are analyzed, and the need for an improved methodology for evaluating the impacts of water use on life cycle bases. Quicklime production is a high energy consumer that is also characterized by high CO2 emissions. This work sets out to develop the energetic and exergetic balance of the limestone calcination process in order to identify the most influential factors in fuel consumption. The results show that the destruction of exergy during due to the combustion of the fuel and the transfer of heat and moment of the process are the most irreversible processes. The results also show that the energy and the exergy contained in the exhaust gases represent the main loss of the process. The combination of these factors represents more than $50 \%$ of the energy supplied to the process.
\end{abstract}

Keywords-- Energy; exergy; efficiency 


\section{INTRODUCCIÓN}

El óxido de calcio $(\mathrm{CaO})$, conocido como cal viva, es uno de los productos alcalinos más empleados en la industria [1]. La producción de cal viva requiere de elevados consumos energéticos e implica importantes emisiones de $\mathrm{CO}_{2}$, actor principal del cambio climático. Otra característica importante es que el consumo de energía requerido por el proceso productivo representa $\approx 50 \%$ de los costos de producción [2].

La evaluación de la eficiencia energética del proceso de producción de la cal en hornos industriales ha sido objeto de varios estudios [2], [3], [4], [5], [6], [7]. La optimización de la operación de los hornos de producción de cal también ha sido estudiada [2], [8]. Ochoa et al. [2] propone un método de optimización experimental mediante métodos estadísticos. ShengXian [8] propone un sistema de diagnóstico basado en el monitoreo holográfico y la simulación en tiempo real del proceso. Este método combina mediciones on-line con la simulación de la calcinación de calizas. Con su aplicación se alcanza un aumento del $25 \%$ en la producción de cal en un horno de prueba.

De acuerdo con Sogut [9] la eficiencia energética es generalmente un componente importante y de bajo costo económico en la estrategia ambiental de una empresa. El análisis energético se fundamenta en el 1er principio de la termodinámica y presenta algunas limitaciones inherentes [10]. Para superar estas limitaciones es necesario incluir en el análisis el $2^{\text {do }}$ principio de la termodinámica. El análisis exergético vincula el $1^{\mathrm{er}}$ y el $2^{\mathrm{do}}$ principio de la termodinámica en el análisis de sistemas térmicos. Utlu et al. [11] señala que comprender las implicaciones exergéticas de un proceso permite una mejor comprensión de la eficiencia y la sostenibilidad. El análisis exergético permite identificar las oportunidades de ahorro más importantes en equipos y procesos térmicos [12].

A pesar de las investigaciones realizadas sobre la evaluación de la eficiencia térmica de hornos para la producción de cal, solo un trabajo [7] utiliza el método exergético. Este trabajo discute algunas implicaciones exergéticas del proceso. No obstante, es necesaria una discusión más profunda sobre los factores que afectan la eficiencia exergética.

El objetivo de este trabajo es evaluar energética y exergéticamente la calcinación de calizas en hornos de cuba vertical e identificar los factores que más influyen sobre la eficiencia térmica del proceso.

\section{DesCRIPCIÓN DEL PROCESO}

Para producir $\mathrm{CaO}$ es necesario calcinar la roca caliza mediante un suministro de calor. El término calcinación se refiere a la disociación del $\mathrm{CaCO}_{3}$ como muestra la reacción (1):

$$
\mathrm{CaCO}_{3}+\text { Calor }=\mathrm{CaO}+\mathrm{CO}_{2}
$$

Está es una reacción altamente endotérmica que requiere $1784 \mathrm{~kJ}$ por $\mathrm{kg}$ de $\mathrm{CaCO}_{3}$. En hornos de cuba vertical esta reacción comienza a $\approx 820^{\circ} \mathrm{C}[13]$, [14], pero en general la reacción se desarrolla a $900^{\circ} \mathrm{C}$ [14].

Existen diferentes diseños de hornos para la calcinación de calizas siendo el horno de cuba vertical el más utilizado por su eficiencia térmica. Un horno de cuba vertical (Fig. 1) es un reactor de lecho móvil con un flujo de gases calientes que ascienden a contracorriente con el flujo de caliza que desciende mientras se calcina a temperaturas que superan los $900^{\circ} \mathrm{C}$. Para una mejor comprensión el horno se divide en tres zonas: precalentamiento, calcinación y enfriamiento. La zona de precalentamiento comienza en la parte superior del horno, donde se suministra la roca caliza y salen los gases de escape. Esta zona termina cuando la roca caliza alcanza la temperatura de reacción y comienza la calcinación. En este punto comienza la zona de calcinación que termina en el nivel de los quemadores donde se suministra el combustible. En esta zona, además de la calcinación, tiene lugar la combustión del combustible. La zona de enfriamiento comienza donde termina la zona de calcinación y termina en el fondo del horno donde se suministra el aire secundario y se extrae la cal viva. El calor requerido para la calcinación se suministra mediante la combustión del combustible adecuado (carbón, petróleo, gas natural, etc.).

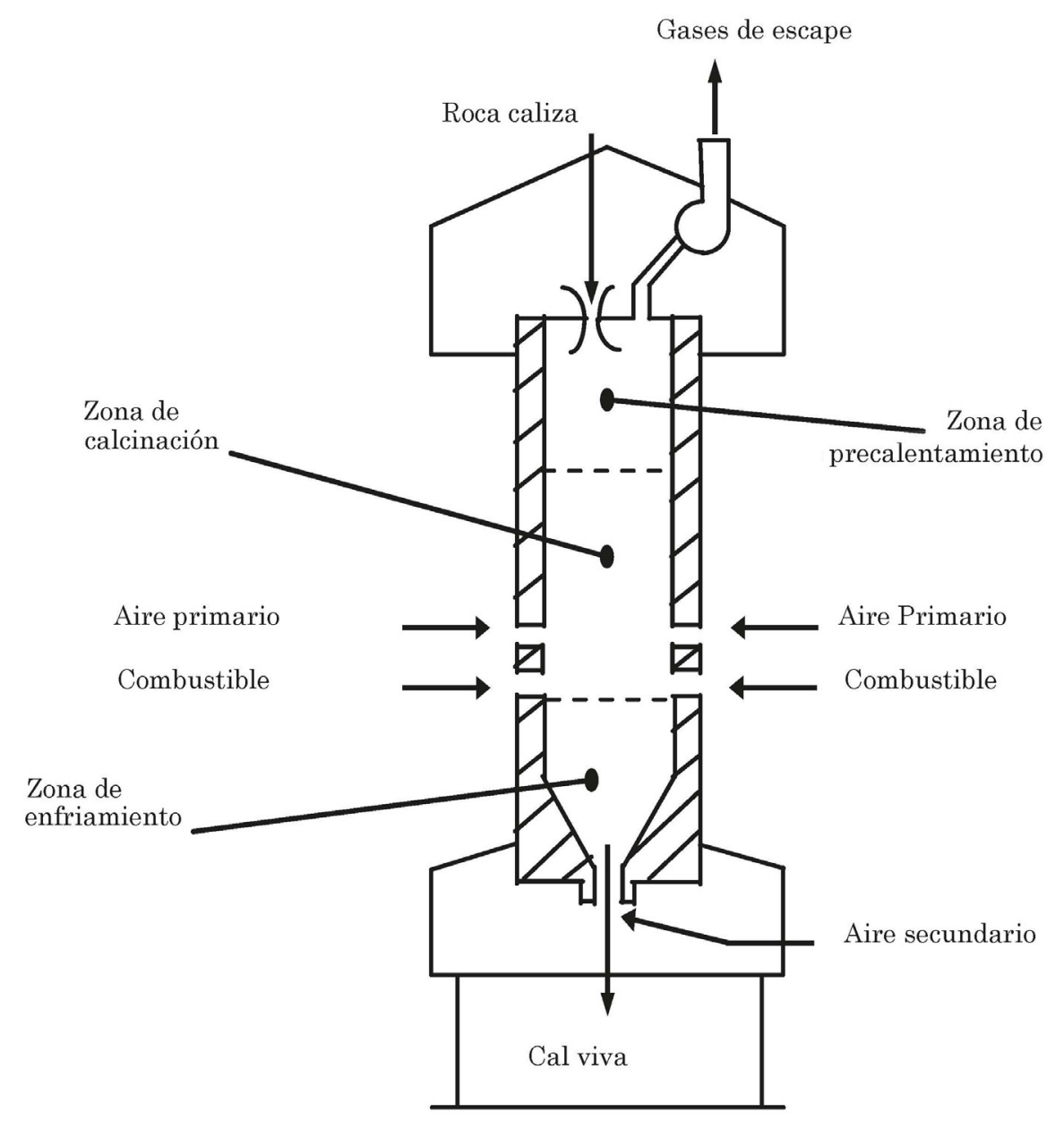

Fig. 1. Horno de cuba vertical. 


\section{MÉTodos DE ANÁLISIS}

El horno se evaluará utilizando el método energético y exergético. Para los cálculos, como el horno es un sistema abierto, se asume que:

- El sistema opera en estado estacionario;

- La energía cinética y potencial del lecho de roca y del gas es despreciable en comparación con su entalpía.

El balance de energía de un sistema abierto (2) está dado por:

$$
\Sigma_{\mathrm{i}} \mathrm{En}_{\mathrm{in}, \mathrm{I}}=\Sigma_{\mathrm{j}} \mathrm{En}_{\text {out.j }}
$$

Donde $\operatorname{En}_{\text {in }}$ representa los flujos de energía a la entrada del proceso y $\mathrm{En}_{\text {inout }}$ representa los flujos de energía a la salida del proceso. El flujo de energía [15] se calcula como (3):

$$
\mathrm{En}_{\mathrm{i}}=\mathrm{m}_{\mathrm{i}} \cdot \mathrm{c}_{\mathrm{pi}} \cdot \Delta \mathrm{T}
$$

Donde $_{\text {cp }}$ es el calor específico y T es la temperatura del flujo i.

Para las propiedades termodinámicas de la roca caliza, la cal viva y los gases se asume el valor correspondiente a la temperatura de entrada a cada zona del horno [8]. La eficiencia energética del horno [3] es igual a (4):

$$
\eta=\frac{H_{R} \cdot A_{q}}{m_{f} \cdot L H V}
$$

El balance exergético del sistema (5) es igual a:

$$
\Sigma_{\mathrm{i}} \mathrm{E}_{\mathrm{in}, \mathrm{i}}=\Sigma_{\mathrm{j}} \mathrm{E}_{\text {out } \mathrm{j}}+\mathrm{E}_{\mathrm{D}}
$$

Donde $\mathrm{E}_{\text {in }}$ es la exergía de los flujos de entrada, $\mathrm{E}_{\text {inou }}$ es la exergía de los flujos de salida y $\mathrm{E}_{\mathrm{D}}$ es la exergía destruida en el proceso.

La exergía del calor [15] es igual a (6):

$$
E_{Q}=\left(1-\frac{T_{o}}{T}\right) \cdot Q
$$

La destrucción de exergía, de acuerdo con la ley de Gouy-Stodola [15], está dada por (7):

$$
E_{D}=T_{0} \cdot S_{\text {gen }}
$$

Donde ${ }_{\text {Sgen }}$ representa la generación de entropía. Para calcular la eficiencia exergética de la producción de cal se considera la exergía química de la cal como producto exergético del proceso (8):

$$
\eta_{e}=\frac{E_{C a O}^{c h}}{E_{F}}
$$

Donde $\mathrm{E}_{\mathrm{F}}$ es la exergía suministrada para desarrollar el proceso.

\section{ResUltados y DISCUSIÓN}

En esta sección se discuten los balances de energía y exergía de dos hornos de cuba vertical. Un horno de cuba vertical usualmente trabaja ininterrumpidamente por varios meses al año. En este caso se midió la operación de un horno durante 24 h, el promedio de los valores medidos en este periodo se considera para los cálculos. Para comparar se considera el horno de cuba vertical discutido por Piringer y Werner [18]. Para diferenciar, los hornos serán referidos como horno No.1 y horno No. 2.

\section{A. Balance de masa}

El balance de masa de un horno de cuba vertical incluye los flujos de roca caliza, combustible y aire y los flujos de salida de cal viva y gases de escape (9):

$$
\mathrm{m}_{\mathrm{Ls}}+\mathrm{m}_{\mathrm{F}}+\mathrm{m}_{\mathrm{A}}=\mathrm{m}_{\mathrm{Ql}}+\mathrm{m}_{\mathrm{G}}
$$

Donde $\mathrm{m}_{\mathrm{Ls}}, \mathrm{m}_{\mathrm{F}}, \mathrm{m}_{\mathrm{A}}, \mathrm{m}_{\mathrm{Ql}} \mathrm{y} \mathrm{m}_{\mathrm{G}}$ representan la masa de roca caliza, combustible, aire, cal viva y gases de escape.

Usualmente una fracción de la roca caliza no disocia durante el proceso. El grado de conversión de la roca caliza (fracción de roca que disocia) puede calcularse en función del \% de $\mathrm{CaO}$ (Aq) [2] contenido en la cal viva (10):

$$
X=\frac{A_{q}}{B \cdot\left(0.44 \cdot A_{q}-0.56\right)}
$$

Donde $\mathrm{B}$ es el \% de $\mathrm{CaCO}_{3}$ contenido en la roca caliza. Considerando la estequiometria de la disociación del $\mathrm{CaCO}_{3}$ (1) y el grado de conversión de la reacción, la masa de cal viva es igual a la diferencia entre la masa de roca caliza alimentada al horno y la masa de $\mathrm{CO}_{2}$ liberada durante la disociación (11):

$$
\mathrm{m}_{\mathrm{Q} 1}=\mathrm{m}_{\mathrm{Ls}} \cdot \mathrm{B} \cdot(1-0.44 \cdot \mathrm{X})
$$

La masa de gases de escapa es igual a (12)

$$
\mathrm{m}_{\mathrm{G}}=\mathrm{m}_{\mathrm{F}}+\mathrm{m}_{\mathrm{A}}+0.44 \cdot \mathrm{X} \cdot \mathrm{B} \cdot \mathrm{m}_{\mathrm{Ls}}
$$

Donde el último término de la ecuación es el $\mathrm{CO}_{2}$ liberado durante la reacción. La Tabla 1 resume el balance de masa para los hornos No. 1 y No. 2. 
Tabla 1. Balance de masa

\begin{tabular}{|l|l|l|l|l|l|}
\hline \multicolumn{3}{|c|}{ Flujos de entrada $(\mathrm{kg} / \mathrm{h})$} & \multicolumn{3}{c|}{ Flujos de salida $(\mathrm{kg} / \mathrm{h})$} \\
\hline & $\begin{array}{l}\text { Horno } \\
\text { No. } 1\end{array}$ & $\begin{array}{l}\text { Horno } \\
\text { No. } 2\end{array}$ & & $\begin{array}{l}\text { Horno } \\
\text { No. } 1\end{array}$ & $\begin{array}{l}\text { Horno } \\
\text { No. } 2\end{array}$ \\
\hline Caliza & 3277 & 14500 & Cal viva & 1965 & 8333 \\
\hline Aire & 3707 & 15875 & Gases & 5249 & 23484 \\
\hline Combustible & 230 & 1692 & Polvo & - & 250 \\
\hline Total & 7214 & 32067 & Total & 7214 & 32067 \\
\hline
\end{tabular}

\section{B. Balance de energía}

El balance de energía de un horno de cuba vertical considera los flujos de entrada de roca caliza, combustible y aire y los flujos de salida de cal viva, gases de escape, entalpía de reacción (calcinación) y pérdidas de calor por las paredes del horno (13):

$$
\mathrm{En}_{\mathrm{Ls}}+\mathrm{En}_{\mathrm{F}}+\mathrm{En}_{\mathrm{A}}=\mathrm{En}_{\mathrm{Ql}}+\mathrm{En}_{\mathrm{G}}+\mathrm{En}_{\mathrm{D}}+\mathrm{En}_{\text {loss }}
$$

La energía suministrada al proceso por el combustible (14) es igual a:

$$
\mathrm{En}_{\mathrm{F}}=\mathrm{m}_{\mathrm{F}} \cdot \mathrm{PCI}
$$

Donde PCI es el poder calorífico inferior del combustible.

La roca caliza y el aire entran al horno a temperatura ambiente. Las temperaturas de los gases de escape y de la de cal viva se calculan en el balance de energía. La temperatura de los gases de escape se calcula según el balance de energía de la zona de precalentamiento [19].

$$
\mathrm{m}_{\mathrm{Ls}}+\mathrm{c}_{\mathrm{pLs}} \cdot\left(\mathrm{T}_{\mathrm{d}}-\mathrm{T}_{\mathrm{o}}\right)+\mathrm{Q}_{\text {loss } \mathrm{PH}}=\mathrm{m}_{\mathrm{g}} \cdot \mathrm{c}_{\mathrm{pg}} \cdot\left(\mathrm{T}_{\mathrm{g}}-\mathrm{T}_{\mathrm{go}}\right)
$$

Donde $\mathrm{T}_{\mathrm{d}}$ es la temperatura de disociación, $\mathrm{T}_{\mathrm{o}}$ es la temperatura ambiente y $\mathrm{Q}_{\text {loss-PHm }}$ es el calor perdido a través de las paredes del horno en la zona de precalentamiento. De acuerdo con [8] las pérdidas de calor en la zona de precalentamiento representan alrededor del $2 \%$ del intercambio total de energía en la zona. De esta forma (15) queda igual a (16):

$$
\mathrm{m}_{\mathrm{Ls}}+\mathrm{c}_{\mathrm{pLS}} \cdot\left(\mathrm{T}_{\mathrm{d}}-\mathrm{T}_{\mathrm{o}}\right)=0.98 \cdot \mathrm{m}_{\mathrm{g}} \cdot \mathrm{c}_{\mathrm{pg}} \cdot\left(\mathrm{T}_{\mathrm{g}}-\mathrm{T}_{\mathrm{go}}\right)
$$

La zona de precalentamiento termina cuando la roca alcanza $900^{\circ} \mathrm{C}$. La temperatura del gas al final de la zona de precalentamiento es entre 1 y $5^{\circ} \mathrm{C}$ mayor que la temperatura de la roca [19].

La temperatura de salida de la roca caliza se calcula considerando la transferencia de calor entre la cal viva y el aire en la zona de enfriamiento [19]:

$$
\begin{aligned}
& m_{Q l} \cdot c_{p Q l} \cdot \frac{d T_{Q l}}{d z}=h \cdot A \cdot\left(T_{Q l}-T_{a}\right) \\
& m_{a} \cdot c_{p a} \cdot \frac{d T_{a}}{d z}=h \cdot A \cdot\left(T_{Q l}-T_{a}\right)
\end{aligned}
$$

Donde $\mathrm{h}$ es el coeficiente de transferencia de calor por convención y A es el área de transferencia de calor de las rocas. La Tabla 2 muestra los resultados del balance de energía.

La fuente principal de energía del proceso es el combustible con más del $98 \%$ de la energía que entra al proceso en ambos hornos. Los diagramas de flujo de la energía para ambos hornos se muestran en la Fig. 2 y Fig. 3. Ambos diagramas se basan en el balance de energía mostrado en la Tabla 2.

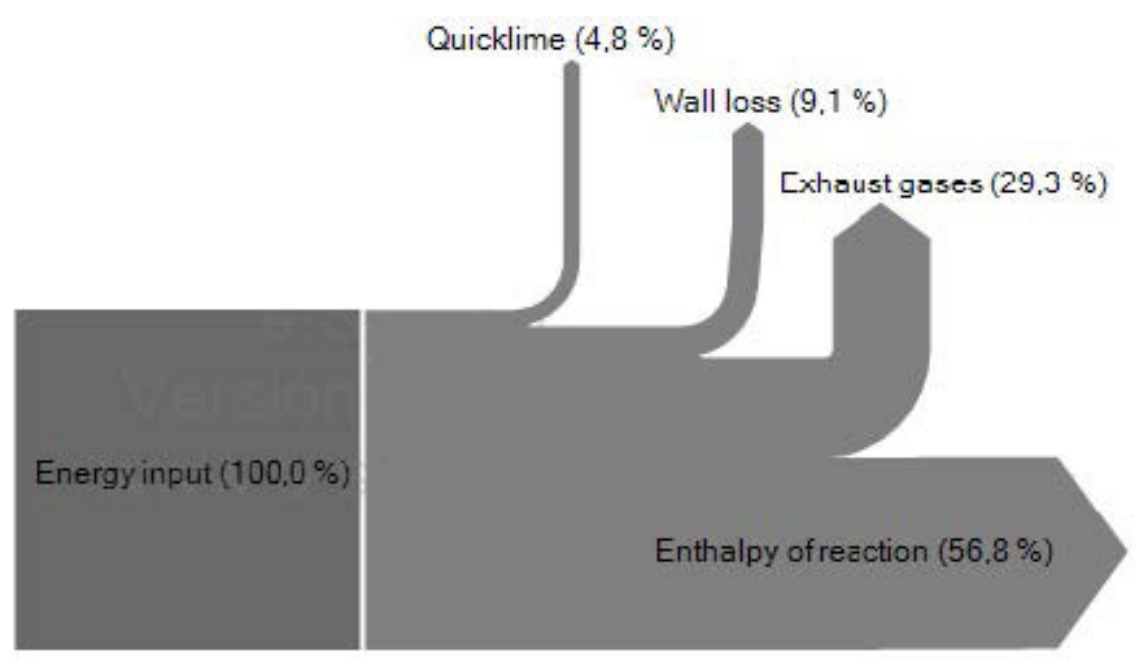

Fig. 2. Balance de energía para el horno No.1.

Tabla 2. Balance de energía

\begin{tabular}{|l|l|l|l|l|l|l|l|l|l|}
\hline \multicolumn{5}{|c|}{ Flujos de entrada } & \multicolumn{6}{c|}{ Flujos de salida } \\
\hline & & Horno No. 1 & & Horno No. 2 & & Horno No. 1 & & Horno No. 2 & \\
\hline & T $(\mathrm{K})$ & En $(\mathrm{kW})$ & $\mathrm{T}(\mathrm{K})$ & En $(\mathrm{kW})$ & & T $(\mathrm{K})$ & En $(\mathrm{kW})$ & $\mathrm{T}(\mathrm{K})$ & En $(\mathrm{kW})$ \\
\hline Caliza & 298 & 21 & 288 & 56 & Cal viva & 550 & 124 & 308 & 66 \\
\hline Aire & 298 & 27 & 294 & 110 & Gases & 728 & 763 & 588 & 2383 \\
\hline Combustible & 298 & 2555 & 293 & 10111 & Entalpia de reacción & - & 1478 & - & 7358 \\
\hline & & & & & Pérdida por paredes & - & 292 & - & 470 \\
\hline Total & - & 2603 & - & 10277 & Total & - & 2603 & - & 10277 \\
\hline
\end{tabular}


Tabla 3. Balance de exergía de los hornos No. 1 y No. 2.

\begin{tabular}{|l|l|l|l|l|l|l|l|l|l|}
\hline & \multicolumn{2}{|c|}{$\begin{array}{l}\text { Horno No. } \\
1\end{array}$} & \multicolumn{2}{|c|}{ Horno No. 2 } & & \multicolumn{2}{|c|}{ Horno No. 1 } & \multicolumn{2}{c|}{ Horno No .2 } \\
\hline $\begin{array}{l}\text { Fuente } \\
\text { entrada }\end{array}$ & $\mathrm{T}$ & $\begin{array}{l}\mathrm{E} \\
(\mathrm{kW})\end{array}$ & $\mathrm{T}$ & $\begin{array}{l}\mathrm{E} \\
(\mathrm{kW})\end{array}$ & $\begin{array}{l}\text { Fuente } \\
\text { salida }\end{array}$ & $\mathrm{T}(\mathrm{K})$ & $\mathrm{E}(\mathrm{kW})$ & $\mathrm{T}(\mathrm{K})$ & $\mathrm{E}(\mathrm{kW})$ \\
\hline Caliza & 298 & 164 & 288 & 725 & Cal viva & 550 & 1114 & 308 & 4844 \\
\hline Aire & 298 & 50 & 296 & 390 & Gases & 728 & 419 & 588 & 1320 \\
\hline Combustible & 298 & 2709 & 293 & 10698 & $\begin{array}{l}\text { Pérdidas } \\
\text { por paredes }\end{array}$ & & 217 & & 158 \\
\hline Total & - & 2977 & & 11814 & Total & & 1750 & & 6322 \\
\hline
\end{tabular}

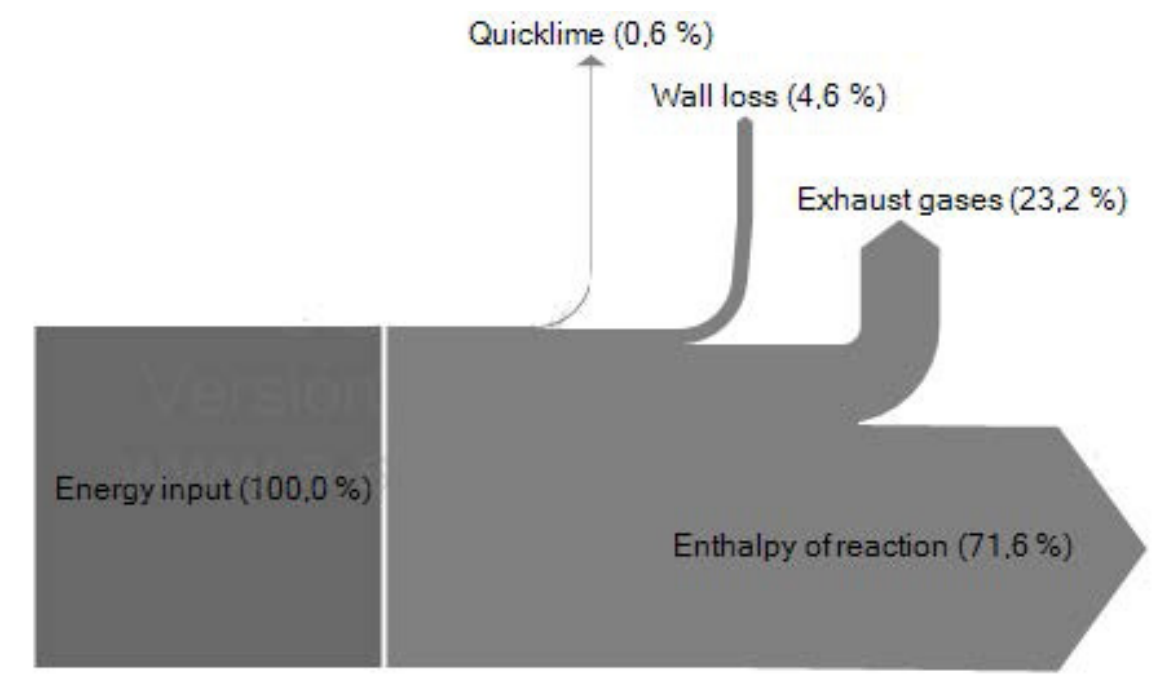

Fig. 3. Balance de energía para el horno No. 2.

El horno No. 2 muestra una mayor eficiencia que el horno No. 1. No obstante, se pueden implementar algunas mejoras en el horno No. 2 reduciendo la temperatura de los gases de escape. Según Bes [19], la temperatura de los gases de escape en un horno que opera de forma óptima es de $150^{\circ} \mathrm{C}$ y la temperatura de la cal viva a la salida del horno es menor a $100^{\circ} \mathrm{C}$.

Un factor que afecta la eficiencia energética es el coeficiente de exceso de aire [19], [20]. Por una parte, incrementar el coeficiente de exceso de aire disminuye las temperaturas del horno lo que lleva a una disminución del ritmo de transferencia de calor y por consiguiente del grado de conversión de la reacción. Para no afectar el grado de conversión con el incremento del coeficiente de exceso de aire es necesario aumentar el consumo de combustible. En ambos casos se afecta negativamente la eficiencia energética. Por otra parte, reducir el coeficiente de exceso de aire impactará la eficiencia de la combustión, reduciendo la energía liberada en el horno. Esto también afectaría el grado de conversión de la calcinación o, para no afectar el grado de conversión, implicaría un aumento del consumo de combustible. Por consiguiente, la selección adecuada del coeficiente de exceso de aire para la combustión es un elemento fundamental para garantizar la eficiencia óptima de la calcinación de calizas. Por otra parte, precalentar el aire para la combustión también puede mejorar la eficiencia energética del proceso.

\section{Balance exergético}

El balance de exergía del horno considera los flujos de entrada de roca caliza, combustible y aire y los flujos de salida de cal viva, gases de escape y pérdidas por paredes (19):

$$
\mathrm{E}_{\mathrm{Ls}}+\mathrm{E}_{\mathrm{F}}+\mathrm{E}_{\mathrm{A}}=\mathrm{E}_{\mathrm{Ql}}+\mathrm{E}_{\mathrm{G}}+\mathrm{E}_{\text {loss }}
$$

La exergía química del combustible [21] es igual a (20):

$$
e_{\mathrm{ch}}^{\mathrm{o}} \cdot=\phi L H V
$$

Donde $\varphi$ es la relación entre la exergía química y el PCI. Para combustibles líquidos está dado por (21):

$$
\varphi=1.0401+0.1728 \cdot \frac{H}{C}+0.0432 \cdot \frac{O}{C}+0.02169 \cdot \frac{S}{C} \cdot\left(1-2.0628 \cdot \frac{H}{C}\right)(21)
$$

Para combustible sólidos es igual a (22):

$$
\varphi=1.0437+0.1896 \cdot \frac{H}{C}+0.0617 \cdot \frac{O}{C}+0.0428 \cdot \frac{N}{C}
$$

Donde C, H, O y S representan la fracción másica del carbono, el hidrógeno, el oxígeno y el azufre del combustible. La Tabla 3 muestra el balance de exergía de los hornos No. 1 y No.2.

De los resultados mostrados en la Tabla 3 la destrucción de exergía en los dos hornos es:

Kiln No. 1: ED = EF - Eout

Kiln No. 2: $\mathrm{E}=1227 \mathrm{~kW}$

\section{1) Generación de entropía}

Durante la calcinación de calizas en hornos de cuba vertical se genera entropía, destruyendo parte de la exergía suministrada al proceso. Las fuentes de generación de entropía son: 
1. Reacciones químicas (combustión y calcinación)

2. Transferencia de calor y momento

3. Transferencia de masa

\section{2) Generación de entropía durante la combustión}

En este caso se asume combustión ideal para los cálculos de generación de entropía [15]:

$$
S_{\text {gen }}=\Delta S_{\text {sys }}+\frac{Q_{\text {out }}}{T_{\text {surr }}}(23)
$$

Donde $\Delta S_{s y s}$ es el cambio de entropía del sistema de reacción, $Q_{\text {out }}$ es el calor que se pierde durante la combustión y $T_{\text {surr }}$ es la temperatura de la frontera donde se pierde el calor.

El cambio de entropía del sistema de reacción es igual a la diferencia entre la entropía de productos y reaccionantes [15]:

$$
\Delta \mathrm{S}_{\text {sys }}=\mathrm{S}_{\text {prod }}-\mathrm{S}_{\text {reac }}
$$

Para el horno No. 1 el exceso de aire es 1.15 y la combustión alcanza los $1600^{\circ} \mathrm{C}$. El horno No. 2 opera con un coeficiente de exceso de aire de 1.32 y la combustión también alcanza los $1600^{\circ} \mathrm{C}$. Para las pérdidas de calor de la combustión se asume la diferencia entre la energía liberada en la combustión adiabática y la entalpía de los gases a la temperatura de combustión (27):

$$
\mathrm{Q}_{\text {out }}=\mathrm{m}_{\mathrm{F}} \cdot \mathrm{LHV}-\mathrm{m}_{\mathrm{gcomb}} \cdot \mathrm{c}_{\mathrm{pg}} \cdot \mathrm{T}_{\text {comb }}
$$

Durante la combustión el calor se pierde al entorno inmediato (gases y roca) que se encuentra a temperaturas cercanas a la temperatura de combustión. Por consiguiente, la generación de entropía producto de la pérdida de calor de la combustión se considera $\mathrm{T}_{\text {surr }}=$ Temperatura de Combustión. Finalmente, la generación de entropía durante la combustión en el horno No.1 (28) es igual a:

$$
\mathrm{S}_{\mathrm{gen}}=32.08\left(\mathrm{~kJ} / \mathrm{kg}_{\mathrm{F}} \cdot \mathrm{K}\right)
$$

Para el horno No. 2 es igual a (29):

$$
\mathrm{S}_{\mathrm{gen}}=21.78\left(\mathrm{~kJ} / \mathrm{kg}_{\mathrm{F}} \cdot \mathrm{K}\right)
$$

\section{3) Generación de entropía en la calcinación}

La disociación del $\mathrm{CaCO}_{3}$ tiene lugar a $900^{\circ} \mathrm{C}$ como se explicó anteriormente. La generación de entropía se calcula según (25). El cambio de entropía del sistema de reacción es igual a (30):

$$
\Delta \mathrm{S}_{\mathrm{sys}}=380.70-214.76=165.94(\mathrm{~kJ} / \mathrm{kmol} \cdot \mathrm{K})
$$

La generación de entropía relacionada con las pérdidas de calor en este caso se calcula utilizando la entalpía y la temperatura de reacción (31):

$$
S_{\text {loss }}=\frac{-Q_{d}}{T_{d}}=-152.08(\mathrm{~kJ} / \mathrm{kmol} \cdot \mathrm{K})
$$

Finalmente, la entropía generada durante la calcinación (32) es igual a:

$$
\mathrm{S}_{\mathrm{gen}}=13.86(\mathrm{~kJ} / \mathrm{kmol} \cdot \mathrm{K})
$$

La generación en este caso se debe a la transferencia de masa del $\mathrm{CO}_{2}$ desde el frente de reacción hacia el flujo de gases. La generación queda igual a (33):

$$
S_{g e n}=m_{\mathrm{CO}_{2}} \cdot\left[c_{P_{\mathrm{CO}_{2}}} \cdot \ln \left(\frac{T_{g}}{T_{d}}\right)-R \cdot \ln \left(\frac{p_{\mathrm{CO}_{2}}}{p_{f}}\right)\right]
$$

La presión en el frente de reacción [22] es igual a (34):

$$
p_{f}=2.5 \cdot 10^{12} \cdot \exp \left(-\frac{168000}{R \cdot T_{d}}\right)(P a)
$$

De acuerdo con la ley de Dalton, la presión de $\mathrm{CO}_{2}$ en el flujo de gases (35) es igual a:

$$
\mathrm{P}_{\mathrm{CO} 2}=\mathrm{X}_{\mathrm{CO} 2} \cdot \mathrm{P}_{\mathrm{TOTAL}}
$$

Donde $\mathrm{x}_{\mathrm{CO} 2}$ es la fracción molar del $\mathrm{CO}_{2}$ en el flujo de gases y $\mathrm{p}_{\text {Total }}$ es la presión total del flujo de gases. La concentración de $\mathrm{CO}_{2}$ en la zona de calcinación varia a medida que avanza la calcinación, en este caso se considera el valor medio de la fracción molar. La temperatura de los gases varía considerablemente en la zona de calcinación. La diferencia media de temperatura entre los gases y el frente de reacción puede considerarse como $(\mathrm{Tg}-\mathrm{Td})=150^{\circ} \mathrm{C}$ [13]. El flujo de $\mathrm{CO}_{2}$ del frente de reacción (considerando el grado de conversión y la estequiometria de la reacción) es igual a (36):

$$
\mathrm{m}_{\mathrm{CO} 2}=0.44 \cdot \mathrm{X} \cdot \mathrm{B} \cdot \mathrm{m}_{\mathrm{Ls}}
$$

Finalmente, la generación de entropía producto de la difusión del $\mathrm{CO}_{2}$ (37): 


$$
\mathrm{S}_{\mathrm{gen}}=14.99 \mathrm{~kJ} / \mathrm{kmol} \cdot \mathrm{K}
$$

La generación de entropía provoca la destrucción de la exergía. La destrucción de exergía se calcula según la ley de Gouy- Stodola (14). La destrucción de exergía debido a la transferencia de calor y momento se calcula como la diferencia entre la destrucción total de exergía, calculado en el balance exergético, y la suma de la exergía destruida por las reacciones químicas y la transferencia de masa (38):

$$
E_{H}^{M T D}=E_{D}{ }^{T}-E_{D}^{C o m b}-E_{D}^{C \text { Cal }}-E_{D}^{\text {Mat }}
$$

A continuación, se ofrecen los resultados de un estudio en la producción de etanol. La Tabla 4 muestra los resultados de la destrucción de exergía en los hornos No.1 y No. 2.

Tabla 4. Destrucción de exergía

\begin{tabular}{|l|l|l|l|l|}
\hline & Horno No.1 & & Horno No.2 & \\
\hline $\begin{array}{l}\text { Fuente de la } \\
\text { destrucción de exergía }\end{array}$ & $\begin{array}{l}\text { Destrucción } \\
\text { de exergía } \\
(\mathrm{kW})\end{array}$ & $\%$ & $\begin{array}{l}\text { Destrucción } \\
\text { de exergía } \\
(\mathrm{kW})\end{array}$ & $\%$ \\
\hline Combustión & 611 & 50 & 3050 & 56 \\
\hline Calcinación & 34 & 3 & 160 & 3 \\
\hline Transferencia de masa & 15 & 1 & 200 & 4 \\
\hline $\begin{array}{l}\text { Transferencia de calor y } \\
\text { momento }\end{array}$ & 567 & 46 & 2083 & 38 \\
\hline Total & 1227 & 100 & 5492 & 100 \\
\hline
\end{tabular}

La Tabla 4 muestra que para ambos hornos la exergía destruida durante la combustión es la principal fuente de irreversibilidades del proceso $(>50 \%$ de la destrucción total de exergía). La destrucción de exergía producto de la transferencia de calor y momento es otra fuente importante de irreversibilidades (46\% y $38 \%$ para los hornos No 1 y No.2 respectivamente). Estos dos procesos representan más del $90 \%$ de las irreversibilidades totales. La exergía destruida producto de la calcinación y transferencia de masa contribuyen con menos del 10\% de la destrucción de exergía.

Otro aspecto que influencia la eficiencia exergética son las pérdidas al entorno. La Fig. 4 y Fig. 5 muestran el balance energético (diagrama de Grassman) de los hornos considerando tanto la exergía destruida como la exergía pérdida al ambiente.

De la Fig. 4 y Fig. 5 la eficiencia exergética del horno No.1 es $36.5 \%$ y para el horno No. 2 es $40.8 \%$. Del balance exergético se concluye que los factores más influyentes sobre la eficiencia exergética de un horno son: destrucción de exergía durante la combustión, destrucción de exergía producto de la transferencia de calor y momento y la pérdida de exergía con los gases

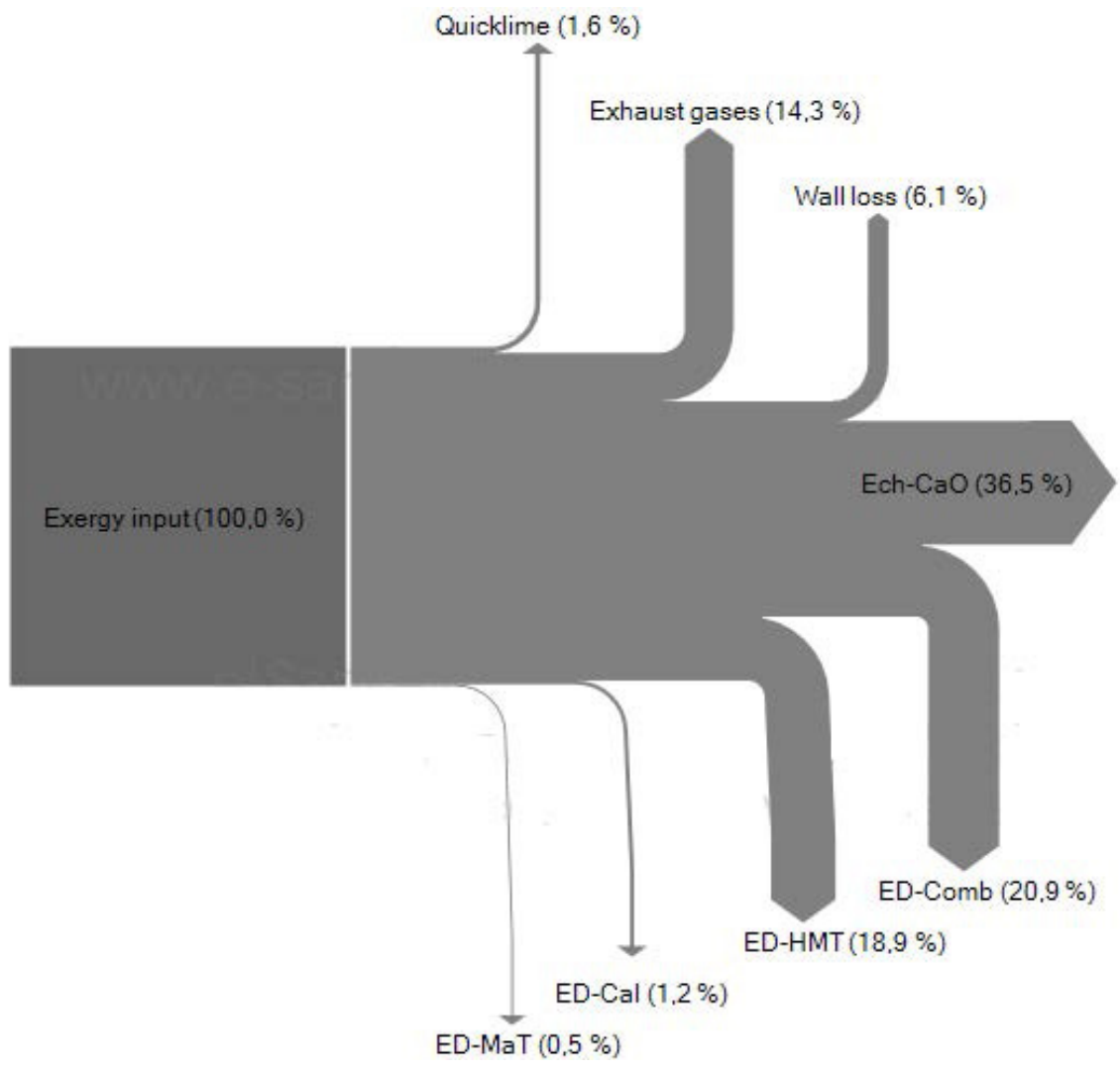

Fig. 4. Balance de exergía para el horno No.1.

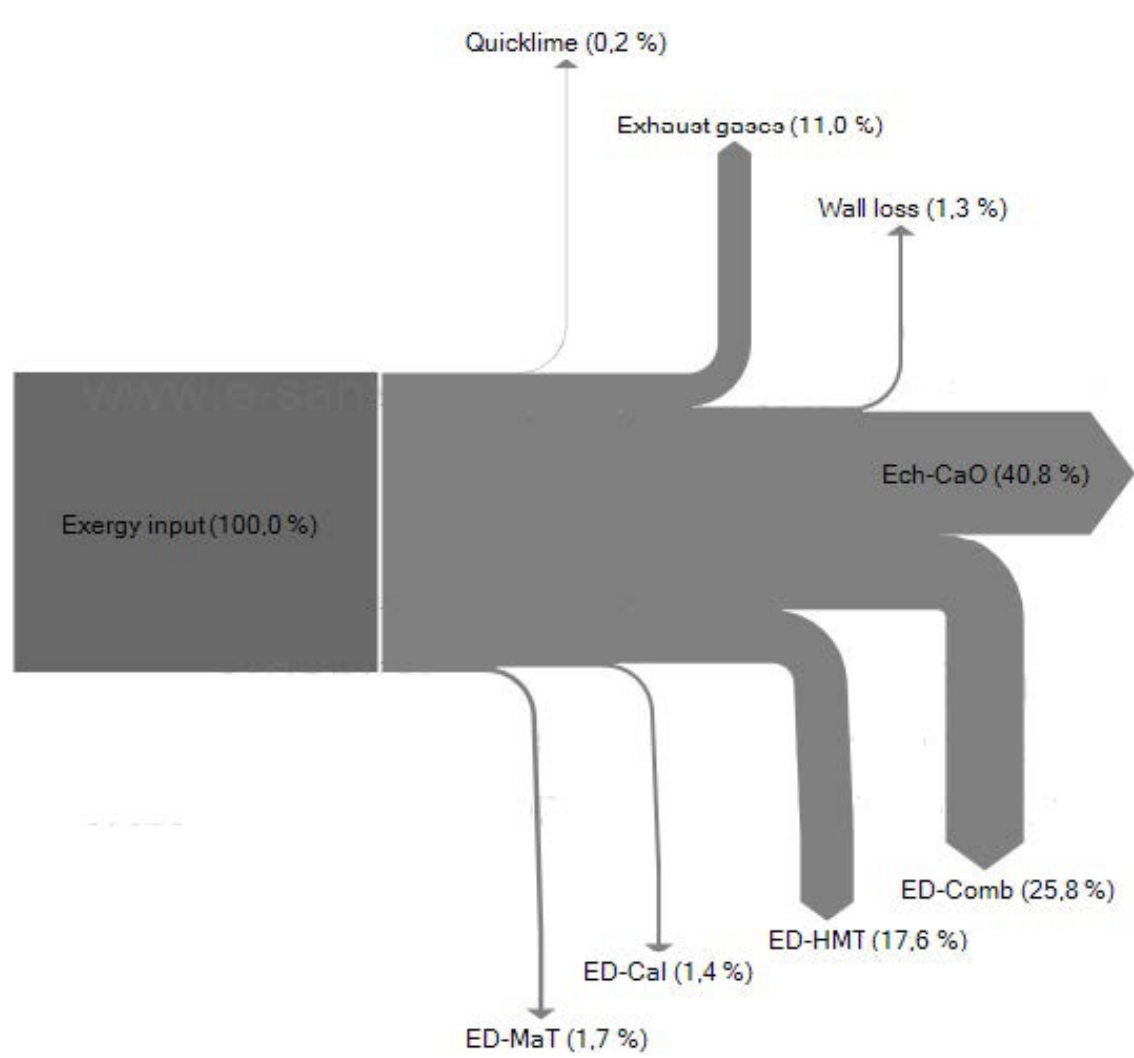

Fig. 5. Balance de exergía para el horno No. 2.

de escape. Estos aspectos son controlados mediante parámetros de operación:

1. Relación caliza/combustible: define la eficiencia del proceso y el valor de otros parámetros de operación. Esta relación se selecciona para garantizar la mejor calidad del producto final con el menor consumo de combustible posible.

2. Exceso de aire de la combustión: incrementar el coeficiente de exceso de aire reducirá el grado de conversión de la calcinación o, para no afectar el grado de conversión, aumentará el consumo de energía. Por otra parte, reducir el coeficiente de exceso de aire afecta la eficiencia de la combustión llevando a los mismos resultados (reducir el grado de conversión o aumentar el consumo de energía). En ambos 
casos se afecta negativamente el consumo específico de combustible de la calcinación lo que disminuye tanto la eficiencia energética como la eficiencia exergética.

3. Dimensiones y distribución de dimensiones de la roca alimentada al horno: el consumo específico de combustible para la calcinación depende en gran medida de las dimensiones de la roca. Las dimensiones y la distribución de dimensiones también afectan la caída de presión en el lecho de rocas. Tanto la caída de presión en el lecho como el consumo específico de combustible afectan el ritmo de transferencia de calor en el horno, lo que a su vez influye sobre la eficiencia térmica [4].

4. Temperatura del flujo de cal viva: la temperatura de salida de la cal viva caracteriza el precalentamiento del aire secundario en la zona de enfriamiento. El funcionamiento óptimo de la zona de enfriamiento influye positivamente en la eficiencia del consumo de combustible [7].

\section{Conclusiones}

Este estudio muestra que la eficiencia de la calcinación de calizas en un horno de cuba vertical es influenciada fundamentalmente por:

1. La destrucción de exergía producto de la combustión.

2. La destrucción de exergía producto de la transferencia de calor y momento.

3. La pérdida de exergía con los gases de escape.

Estos factores representan más del 50\% de la exergía destruida o pérdida en el proceso y están influenciados fundamentalmente por el tipo de combustible, el tipo de horno y su tecnología y los parámetros de operación. la vía más rápida y económica de mejorar la eficiencia del horno es controlando los parámetros de operación.

La vía más rápida y económica de mejorar la eficiencia del horno es controlando los parámetrso de operación. Los parámetros más importantes son: relación caliza/combustible, coeficiente de exceso de aire de la combustión, dimensión y distribución de dimensiones de la roca alimentada al horno y la temperatura del flujo de cal viva. La eficiencia óptima del horno depende de la combinación óptima de los parámetros de operación más que del valor óptimo de cada parámetro individual. para identificar la combinación óptima de los parámetros de operación es importante identificar la destrucción evitable e inevitable de exergía en el proceso. Esto garantizará una eficiencia óptima y un producto final con la calidad requerida.

Optimizar la eficiencia de la calcinación de calizas además contribuye a disminuir las emisiones de $\mathrm{CO}_{2}$ asociadas al proceso.

\section{REFERENCIAS}

[1] A. Sagastume, J. Van Caneghem, J. B. Cogollos and C. Vandecasteele, "Evaluation of the environmental performance of lime production in Cuba", J Clean Prod, vol. 31, no. 1, pp. 126-136, Aug. 2012. https://doi.org/10.1016/j. jclepro.2012.02.035

[2] P. A. Ochoa, A. Sagastume, J. B. Cogollos and C. Vandecasteele, "Cleaner production in a small lime factory by means of process control", J Clean Prod, vol. 18, no. 12, pp. 1171-1176, Aug. 2010. https://doi.org/10.1016/j.jclepro.2010.03.019

[3] R. S. Boynton, Chemistry and Technology of Lime and Limestone, New York: John Wiley \& Sons, 1980.

[4] H. Ruch, "The theoretical limits of heat consumption in lime burning due to the physical and chemical laws", $Z K G$ Int, vol. 34, no. 1, pp. 20-26, Jan, 1981.

[5] Y. De la Peña, G. Bordeth; H. Campo; \& U. Murillo "Clean Energies: An Opportunity to Save the Planet", IJMSOR, vol. 3, no. 1, pp. 21-25, 2018. https://doi.org/10.17981/ijmsor.03.01.04

[6] Duarte Forero, J., Guillín Estrada, W., \& Sánchez Guerrero, J. (2018). Desarrollo de una metodología para la predicción del volumen real en la cámara de combustión de motores diésel utilizando elementos finitos. INGE CUC, 14(1), 122-132. https://doi.org/10.17981/ingecuc.14.1.2018.11.

[7] A. Sagastume and C. Vandecasteele, "Exergy-based indicators to evaluate the possibilities to reduce fuel consumption in lime production", Energy, vol. 36, no. 5, pp. 2820-2827, May. 2011. https://doi.org/10.1016/j.energy.2011.02.023

[8] D. Sheng-xiang, X. Qing-song and Z. Jie-min, "A lime shaft kiln diagnostic expert system based on holographic monitoring and real-time simulation," Expert Syst Apl, vol. 38 , no. 12 , pp. 15400-15408, Nov. 2011. https://doi. org/10.1016/j.eswa.2011.06.021

[9] M.Z. Sogut, Z. Oktay and A. Hepbasli, "Energetic and exergetic assessment of a trass mill process in a cement plant," Energy Convers Manag, vol. 50, no. 9, pp. 2316-2323, Sep. 2009. https://doi.org/10.1016/j.enconman.2009.05.013

[10] P. Regulagadda, I. Dincer and G. F. Naterer, "Exergy analysis of a thermal power plant with measured boiler and turbine losses", Appl Therm Eng, vol. 30, no. 8-9, pp. 970-976, Jun. 2010. https://doi.org/10.1016/j.applthermaleng.2010.01.008

[11] Z. Utlu, Z Sogut, A. Hepbasli and Z. Oktay, "Energy and exergy analyses of a raw mill in a cement production", $A p p l$ Therm Eng, vol. 26, no. 17-18, pp. 2479-2489, Dec. 2006. https://doi.org/10.1016/j. applthermaleng.2005.11.016

[12] I. H. Aljundi, "Energy and exergy analysis of a steam power plant in Jordan", Appl Therm Eng, vol. 29, no. 2-3, pp. 324-328, Feb. 2009. https://doi.org/10.1016/j.applthermaleng.2008.02.029

[13] A. Senegacnik, J. Oman and B. Sirok, "Analysis of calcination parameters and the temperature profile in an annular shaft kiln. Part 1: Theoretical survey", Appl Therm Eng, vol. 27, no. 8-9, pp. 1473-1482, Jun. 2007. https://doi. org/10.1016/j.applthermaleng.2006.09.026

[14] P. L. Zuideveld and P. J. van den Berg, "Design of lime shaft kilns", Chem Eng Sci, vol. 26, no. 6, pp. 875-883, Jun. 1971. https://doi.org/10.1016/0009-2509(71)83048-8

[15] Y. A. Çengel and M. A. Boles, Thermodynamics: An Engineering Approach, 5th ed. Boston, MA, USA; McGrawHill, 2006.

[16] A. Bejan, G. Tsatsaronis and M. J. Moran, Thermal design and optimization. New York: John Wiley \& Sons, 1996. 
[17] C. Koroneos, G. Roumbas and N. Moussiopoulos, "Exergy analysis of cement production", IJEX, vol. 2, no. 1, pp. 5568, Jan. 2005. https://doi.org/10.1504/IJEX.2005.006433

[18] H. Piringer and W. Werner, "Conversion of large-diameter single shaft kilns to lignite dust firing successfully concluded", ZKG Int, vol. 61, no. 1, pp. 46-52, Jan. 2008.

[19] A. Bes, "Dynamic Process Simulation of Limestone Cal cination in Normal Shaft Kilns", Doctoral Thesis, ISUT, Magdeburg, de, 2006. Available: www.uni-magdeburg.de/ isut/TV/English/Research/Project/Bes.pdf

[20] A. Bes, E. Specht and G. Kehse, "Calculation of the cooling zone length and the lime discharge temperature of lime shaft kilns", ZKG Int, vol. 60, no. 4, pp. 63-73, Jan. 2007.

[21] T. Kotas, The exergy method of thermal plant analysis, 2th ed., Krieger Publishing Company, Fla, USA, 1995.

[22] C. Chen, E. Specht and J. Kehse, "Influences of origin and material property of limestone upon its decomposition behavior in shaft kilns", $Z K G$ Int, vol. 60, no. 1, pp. 51-60, Jan. 2007 